entries eqnarray

ITEP/TH-77/98

hepth/9812519

\title{
Low energy theorems and the Dirac operator spectral density in QCD
}

\author{
A.Gorsky \\ ITEP, Moscow, 117259, B.Cheryomushkinskaya 25
}

\begin{abstract}
We discuss the behaviour of the spectral density of the massless Dirac operator at the small eigenvalues and quark masses compatible with the restrictions imposed by the low energy theorems in QCD. Sum rule for its derivative over the quark mass is found.
\end{abstract}

1. The search for the universal characteristics of QCD at the strong coupling regime remains the important problem to clarify the structure of QCD vacuum. One of the most important universal objects is the spectral density of the massless Dirac operator whose behaviour near zero eigenvalue provides the pattern of the spontaneous symmetry breaking. The Banks-Casher relation [1] states that the density at the origin is the fermionic condensate $<\bar{q} q>=-\pi \rho(0)$, where

$$
\begin{gathered}
\hat{D} q=\lambda q \\
\rho(\lambda)=<V^{-1} \sum \delta\left(\lambda-\lambda_{n}\right)>_{A}
\end{gathered}
$$

$\mathrm{V}$ is the Euclidean volume and averaging over the gluon ensemble is assumed. Generalization of the Banks-Casher relation for the moments of the density at the finite volume has been found in [4]. The obvious question concerns the behaviour of the spectral density at small masses and eigenvalues. In the perturbation theory density behaves as $\rho(\lambda)=c \lambda^{3}$

therefore the linear and quadratic terms have the nonperturbative nature. It would describe the critical behaviour of the system at zero temperature and possibly fix a universality class.

Let us remark that the universality properties of the spectral density allow to apply the matrix model technique to analyse its behaviour at the finite volume (see 8 ] for the review).

It is natural to relate the characteristics of the spectral density to other universal objects in QCD. The best candidates are the low energy theorems for the zero-momentum QCD correlators in the different channels 
which amount from the chiral Ward identities [2, 3]. The first attempt to get the information about the spectral density behaviour involved isovector scalar correlator $\int d x<S^{i}(x) S^{j}(0)>$ [5.

It was claimed that it yields the term linear in $\lambda$ that vanishes for $N_{f}=2$. Recently two other sum rules arising from the correlators

$$
\int d x<A^{i}(x) A^{j}(0)-V^{i}(x) V^{j}(0)>
$$

[6] and

$$
\int d x<S^{i}(x) S^{j}(0)-\delta^{i j} P^{0}(x) P^{0}(0)>
$$

[7] were obtained but no additional information on the spectral density has been extracted.

In this note we discuss the complete set of restrictions imposed by low energy theorems for the two point and three point correlators on the spectral density. Correlators in the scalar and pseudoscalar channels yield the information on the spectral density itself while those in the vector and axial channels are relevant only for the correlations of the eigenvalues.

2. Let us consider the correlators in the isovector and isoscalar scalar and pseudoscalar channels in the $N_{f}=2$ case. In what follows we shall discuss only correlators which are free from the nonuniversal high energy contributions. The complete list of such low- energy theorems for two, three and four point correlators can be found in [2]. Corresponding correlators can be expressed in terms of the spectral density as follows;

$$
\begin{gathered}
\int d x<\delta^{i j} S^{0}(x) S^{0}(0)-P^{i}(x) P^{j}(0)>=-\frac{G_{\pi}^{2} \delta_{i j}}{M_{\pi}^{2}}+\delta_{i j} \frac{B^{2}}{8 \pi^{2}}\left(l_{3}-4 l_{4}+3\right)= \\
-\delta_{i j}\left(\int \frac{8 m^{2} \rho(\lambda, m)}{\left(\lambda^{2}+m^{2}\right)^{2}}-4 m \int \frac{\partial_{m} \rho(\lambda, m)}{\left(\lambda^{2}+m^{2}\right)}\right) \\
\int d x<S^{i}(x) S^{j}(0)-\delta_{i j} P^{0}(x) P^{0}(0)>=-\delta_{i j} 8 B^{2} l_{7}=- \\
\delta_{i j}\left(\int \frac{8 m^{2} \rho(\lambda, m)}{\left(\lambda^{2}+m^{2}\right)^{2}}-4 \frac{<\int d x Q(x) Q(0)>}{m^{2} V}\right) \\
\int d x<P^{3}(x) P^{0}(0)>=\frac{G_{\pi} \tilde{G}_{\pi}}{M_{\pi}^{2}}= \\
8\left(m_{u}-m_{d}\right) m \int \frac{\rho(\lambda, m)}{\left(\lambda^{2}+m^{2}\right)^{2}}-\frac{4\left(m_{u}-m_{d}\right)<\int d x Q(x) Q(0)>}{m^{3} V}
\end{gathered}
$$

where $G_{\pi}=2 F_{\pi} B=\frac{F_{\pi} m_{\pi}^{2}}{m} ; \tilde{G}_{\pi}=-\left(m_{u}-m_{d}\right) \frac{4 B^{2} l_{7}}{F_{\pi}}$ and $Q(x)$ is the topological charge density. Sum rule arising from the last low energy theorem exactly coincides with

the one coming from correlator (3). Low energy constants $l_{3}, l_{4}$ behave as logm at small quark masses, while the constant $l_{7}$ contains no chiral logarithms [2]. 
We can add here low energy theorem for three point correlator

$$
\begin{gathered}
\int d x d y<S^{0}(x) P^{i}(y) P^{k}(0)>=\frac{\delta_{i k} G_{\pi}^{3}}{M_{\pi}^{4} F_{\pi}}-\delta_{i k} \frac{B^{2} G_{\pi} F_{\pi}}{8 \pi^{2} M_{\pi}^{2}}\left(l_{3}-4 l_{4}+3\right)= \\
\delta_{i j} \frac{d}{d m} \int \frac{2 \rho(\lambda, m)}{\lambda^{2}+m^{2}} .
\end{gathered}
$$

It appears that the sum rules resulted from the low energy theorems (2)

and (5) are identical.

The last low energy theorem which is potentially important amounts from the four point pseudoscalar correlator

$$
\begin{gathered}
\int d x d y d z<P^{i}(x) P^{j}(y) P^{k}(z) P^{l}(0)>=-\frac{G_{\pi}^{4}}{F_{\pi}^{2} M_{\pi}^{8}}\left(\delta_{i j} \delta_{k l}+\delta_{i k} \delta_{j l}+\delta_{i l} \delta_{j k}\right)\left(-\frac{M_{\pi}^{2}}{F^{2}}+\frac{M_{\pi}^{4}\left(24 l_{4}-9\right)}{96 \pi^{2} F^{4}}+\ldots\right)= \\
\left(\delta_{i j} \delta_{k l}+\delta_{i k} \delta_{j l}+\delta_{i l} \delta_{j k}\right) \int \frac{4 \rho(\lambda, m)}{\left(\lambda^{2}+m^{2}\right)^{2}}+R_{i j k l} .
\end{gathered}
$$

Unfortunately it is more difficult to extract the information from the four point correlator since there is the contribution $R_{i j k l}$ corresponding to the diagram with at least two fermionic loops which can,t be expressed in terms of the spectral density. Moreover the sum rules are sensible to the two loop contribution to the four point correlator in the chiral theory which is unknown at a moment. Therefore there are only two rigorous independent sum rules for the spectral density.

Combination of the sum rules (2) and (3) yields the following model independent sum rule for the mass derivative of the spectral density

$$
\begin{gathered}
m \int \frac{4 \partial_{m} \rho(\lambda, m)}{\left(\lambda^{2}+m^{2}\right)}= \\
\frac{B^{2}}{8 \pi^{2}}\left(l_{3}-4 l_{4}+3\right)-\frac{G_{\pi}^{2}}{M_{\pi}^{2}}+8 B^{2} l_{7}+\frac{4<\int d x Q(x) Q(0)>}{m^{2} V} .
\end{gathered}
$$

3. Turn now to the discussion of the restrictions imposed by the sum rules on the behaviour of the density near the origin. We would like to look for the following anzatz

$$
\rho(\lambda, m)=\rho(0)+c_{1} \lambda \log \lambda+c_{2} \lambda+c_{3} m+c_{4} m \log m+c_{5} m \log ^{2} m+O\left(m^{2}, \lambda^{2}, m \lambda\right),
$$

where $c_{i}$ are the constants to be found. Let us first make an assumption that the spectral density has to be regular if $\lambda \rightarrow 0$ or $m \rightarrow 0$ so there are no terms like $\left(\frac{\lambda}{m}\right)^{n}$ or $m \log \lambda$ in the expansion above. It appears that this anzatz is consistent with the low-energy theorems for the correlators which are free from the high energy contributions.

Apart from the sum rules above we assume two additional model independent restrictions on the spectral density. First we use the universality of the mlogm correction to the chiral condensate [3]. Secondly there is unambiguous statement that there are no logm contributions to the correlator $\left\langle S^{j} S^{i}\right\rangle$. This fact has been already used to show that $c_{2}=0$ [5]. 
Consider first correlators $(2),(5)$ which give rise the identical sum rules. The leading $m^{-1}$ singularity immediately comes from $\rho(0)$ term but the matching of the logm term appears to be a subtle point. It is easily seen that the constants $c_{4}, c_{1}$ don,t contribute hence one has to assume that $c_{5} \neq 0$.

The correction to the condensate looks as

$$
<\bar{q} q>_{m}=<\bar{q} q>_{0}\left(1-\frac{3 M_{\pi}^{2} \log M_{\pi}^{2}}{32 \pi^{2} F_{\pi}^{2}}+\ldots\right)
$$

and it is supposed that there is no $m \log ^{2} m$ term. Hence using the Banks-Casher formula for the condensate we can claim that to cancel $m \log ^{2} m$ correction we have to assume that $c_{1} \neq 0$.

However this term in the spectral density yields the divergence in the integral at the UV region rising the question on the substraction of the perturbative contribution. The proper version of this proceedure which would allow to make a prediction for $c_{1}, c_{4}$ deserves further investigation.

To discuss the restriction on $c_{3}$ we have to expand the topological susceptibility up to the second order in the quark mass

$$
\frac{<\int d x Q(x) Q(0)>}{V}=\frac{m B F_{\pi}^{2}}{2}+d B^{2} m^{2}+\ldots,
$$

hence the coefficient $d$ enters our sum rules. If we substitute the expression for the spectral density and assume that all logarithmic contribution cancel the model independent relation arises

$$
c_{3}=-8 l_{7}+2 d .
$$

Note that the analogous relation has been considered in [7] but the term in the lhs has been missed.

Since the constants $c_{1}$ and $c_{5}$ can,t vanish simultaneously the $m^{2} \log m$ and $m^{2} \log ^{2} m$ terms have to be manifest in the expansion of the topological susceptibility. While the logm term can be attributed to the first correction to the condensate the origin of the $\log ^{2} m$ term is unclear. Certainly it is desirable to consider the $N_{f}>2$ case when the dependence of the topological susceptibility on the quark masses is more transparent.

Recently the behaviour of the spectral density at small quark masses with arbitrary number of flavours has been found within the matrix theory approach [9]. It was claimed that the singular terms are present in the diffusive domain where the spectral density manifests the $m \log \lambda$ contribution. It can be shown that such term is consistent with the sum rule found above. However it is not clear how possible singularity agrees with the nonzero quark condensate.

4. In this note we considered the behaviour of the Dirac operator spectral density around the origin. It appeared that the low energy theorems impose strong restrictions on it but don,t determine it unambigously. It would be crusual to derive the complete list of the two loop contributions to the off shell correlators in the chiral theory since this information provides the additional restrictions on the linear terms in the spectral density as well as on the $\lambda^{2}$ terms. We have shown that the nonsingular spectral density manifests unexpected $\operatorname{mlog}^{2} m$ term which possibly can be attributed to the renormalization of the mass in the strong external field similar 
to the QED renormalization of the mass [10]. Otherwise some singular terms [9] in the spectral density have to be admitted.

This work is partially motivated by an attempt to develop the interpretation of the spectral density of the Dirac operator within the brane approach. This issue was addressed for the $\mathrm{N}=1$ SUSY theory in [11] and quark masses fix the positions of D6 branes representing the fundamental matter in the "momentum" space. Therefore to clarify the brane picture it is necessary to elaborate the $N_{f}$ dependence of the spectral density as well the the case of the generic mass matrix. These questions will be discussed elsewhere.

I am grateful to H.Leutwyler for the hospitality in ITP at Bern University and discussions which initiated this work. I would like to thank A.Smilga for the useful comments and D.Toublan for bringing reference [9] to my attention. The work was supported in part by grants INTAS-96-0482 and RFBR 97-02-16131.

\section{References}

[1] T.Banks and S.Casher, Nucl.Phys.B169 (1980) 103

[2] J.Gasser and H.Leutwyler, Ann.Phys.158 (1984) 142

[3] V.Novikov, M.Shifman, A.Vaishtein and V.Zakharov, Nucl.Phys. B191 (1981) 301

[4] H.Leutwyler and A.Smilga, Phys.Rev. D46 (1992) 5607

[5] A.Smilga and J.Stern, Phys.Lett.B318 (1993) 531

[6] J.Stern, hepth/ 9801107

[7] A.Smilga, hepth/9805214

[8] J.J.M.Verbaarschot, hepth/9705125

[9] J.C.Osborn, T.Toublan and J.J.M.Verbaarschot, Nucl.Phys. B540 (1999) 317 ; hepth/9806110

[10] V.P.Gusynin, V.A Miranski and I.A.Shovkovy, Phys.Rev. D52 (1995) 4747, hepph/9501304

V.P.Gusynin and A.V.Smilga hepph/9807486

[11] A.Gorsky, ZHETP. Lett. 69 (1999) 3 ; hepth/9804020 